Original Research Paper

\title{
Partial Eigenvalue Assignment for High-Order Linear Systems in a Time Delayed System
}

\author{
${ }^{1,2}$ Ehab A. El-Sayed and ${ }^{1}$ Eid E. EI Behady \\ ${ }^{I}$ Department of Mathematics, College of Science and Humanitarian Studies, \\ Prince Sattam Bin Abdulaziz University, Al-Kharj, Saudi Arabia \\ ${ }^{2}$ Department of Science and Engineering Mathematics, \\ Faculty of Petroleum and Mining Engineering, Suez University, Egypt
}

\author{
Article history \\ Received: 19-01-2016 \\ Revised: 02-08-2016 \\ Accepted: 29-09-2016 \\ Corresponding Author: \\ Ehab A. El-Sayed \\ Department of Mathematics, \\ College of Science and \\ Humanitarian Studies, Prince \\ Sattam Bin Abdulaziz \\ University, Al-Kharj, Saudi \\ Arabia \\ Email: janine.tiu@gmail.com
}

\section{Introduction}

Consider high order dynamical system (Duan, 2005; Dedieu and Tisseur, 2003; Dedieu and Tisseur, 2001) of the form:

$$
M_{k} \frac{d^{k}}{d t^{k}} v(t)+\ldots+M_{1} \frac{d}{d t} v(t)+M_{0} v(t)=0
$$

where, $\left\{M_{k}, M_{k-1}, \ldots, M_{1}, M_{0}\right\}$ are real symmetric $n \times n$ matrices and $M_{k}$ is positive definite and $v(t)$ is a timedependent $n \times 1$ vector. Using $v(t)=x e^{\lambda t}$ in equation (1.1), where $x$ is a real vector, then:

$$
P(\lambda) x=\left(\lambda^{k} M_{k}+\lambda^{k-1} M_{k-1}+\ldots+\lambda M_{1}+M_{0}\right) x=0
$$

Where matrix polynomial:

$$
P(\lambda)=\lambda^{k} M_{k}+\lambda^{k-1} M_{k-1}+\ldots+\lambda M_{1}+M_{0}
$$

Is very often referred to as $\lambda$ matrix, or matrix polynomial of degree $k$ (Dedieu and Tisseur, 2003; Dedieu and Tisseur, 2001; Wang and Zhang, 2013; 2014; Ramadan and El-Sayed, 2010). lambda $(\lambda)$ is the characteristic roots of Equation (1.3), i.e., $\operatorname{det}(P(\lambda))=0$, are known as eigenvalues. The vectors $y \neq 0$ and $x \neq 0$ are corresponding left and right eigenvectors respectively which satisfy $y^{H} P(\lambda)=0$ and $P(\lambda) x=0$, where $H$ is the conjugate transpose. The standard eigenvalue problem $A x=\lambda x$ is the special case of (1.2) for example, the references Wang and Zhang, 2013; 2014; Ramadan and El-Sayed, 2010).

The system modeled by (1.1) can be controlled with the application of a forcing function $B u(t-\tau), B \in R^{n \times m}$, $(m \leq n)$ ( $B$ the control matrix) and $u(t-\tau)$ is a timedependent $m \times 1$ real vector and $\tau$ is a constant time delay. The matrix $B$ has full column rank, that is, $\operatorname{rank}(B)=m$ in which case (1.1) is replaced by:

$$
\begin{aligned}
& M_{k} \frac{d^{k}}{d t^{k}} v(t)+M_{k-1} \frac{d^{k-1}}{d t^{k-1}} v(t) \\
& +\ldots+M_{1} \frac{d}{d t} v(t)+M_{0} v(t)=B u(t-\tau)
\end{aligned}
$$

With the choice of the controlling force:

$$
\begin{aligned}
& u(t-\tau)=F_{1}^{T} \frac{d^{k-1}}{d t^{k-1}} v(t-\tau) \\
& +\ldots+F_{k-1}^{T} \frac{d}{d t} v(t-\tau)+F_{k}^{T} v(t-\tau)
\end{aligned}
$$

Such that, $F_{1}, F_{2}, \ldots, F_{k} \in R^{n \times m}$ constant matrices, leads to the closed loop system:

$$
\begin{aligned}
& M_{k} \frac{d^{k}}{d t^{k}} v(t)+M_{k-1} \frac{d^{k-1}}{d t^{k-1}} v(t)+\ldots+M_{1} \frac{d}{d t} v(t)+M_{0} v(t) \\
& =B\left(F_{1}^{T} \frac{d^{k-1}}{d t^{k-1}} v(t-\tau)+\ldots+F_{k-1}^{T} \frac{d}{d t} v(t-\tau)+F_{k}^{T} v(t-\tau)\right)
\end{aligned}
$$


Differential Equation (1.6) leads, with the separation of variables $v(t)=x e^{\lambda t}$, to the problem of finding the eigenvalues of the modified matrix polynomial:

$$
\begin{aligned}
& P_{c}(\lambda, \tau)=\lambda^{k} M_{k}+\ldots+\lambda M_{1} \\
& +M_{0}-B\left(F_{1}^{T} \lambda^{k-1}+\ldots+F_{k-1}^{T} \lambda+F_{k}^{T}\right) e^{-\lambda \tau}
\end{aligned}
$$

The partial eigenvalue assignment problem for the time delayed system (1.6) is to find the control gain matrices $F_{1}, F_{2}, \ldots, F_{k}$ such that:

$$
\operatorname{det}\left(P_{c}\left(\lambda_{i}, \tau\right)\right)=0, \text { for } i=1,2, \ldots, k n
$$

has the desired eigenvalues $\left\{\mu_{1}, \quad \mu_{2}, \ldots, \mu_{m}\right.$, $\left.\lambda_{m+1}, \ldots, \lambda_{k n}\right\}$. That it contains $m$ numbers of new assigned desired eigenvalues $\left\{\mu_{1}, \mu_{2}, \ldots, \mu_{m}\right\}$ without affecting the remaining eigenvalues $\left\{\lambda_{m+1}, \ldots, \lambda_{k n}\right\}$ of the Equation (1.1). Throughout this paper, notion (PEAP) is used instead of partial eigenvalue assignment problem.

PEAP arises from many practical situations such as electrical circuit simulation and acoustic system, vibration analysis of structural mechanical, fluid mechanics, finite element model updating in automobile industries and aerospace (Arévalo and Lötstedt, 1995; De Boor and Kreiss, 1986; Sand, 2002; Mackey et al., 2006).

PEAP for high-order linear systems without time delay introduced in many paper (Wang and Zhang, 2014; Ramadan and El-Sayed, 2010). Prattand and Singh (2009) were solution PEAP for second order linear system with known time delay in single-input case. Also Singh et al. (2014) were solution the same problem but in Multi-input case and study its stability with known time delay. Wang and Zhang (2013) were introduced the direct method of PEAP for high order systems in single input case using receptance method with known time delay. In this paper, we proposed a method to solve PEAP for high order control systems in single input case and multi-input case with known time delay, using orthogonality relations between the eigenvectors of matrix polynomial. This solution requires only a partial knowledge of the eigenvalue and the corresponding eigenvector of the matrix polynomial which allows the partial assignment of desired eigenvalues with no spillover. The numerical examples illustrate that the proposed method.

\section{Orthogonality Relations between the Eigenvectors of Matrix Polynomial}

In this section, we introduce some Orthogonality relations which are play role important for solving our problem.
Theorem 1: (Sarkissian, 2001) Orthogonality of the Eigenvectors of a Matrix A

Let $\lambda_{1}, \lambda_{2}, \ldots, \lambda_{n}$ be the eigenvalues of a matrix $A \in C^{n \times n}$ and let $\hat{X}$ and $\hat{Y}$ be respectively the right and the left eigenvector matrices of $A$. Assume that $\left\{\lambda_{1}\right.$, $\left.\lambda_{2}, \ldots, \lambda_{m}\right\} \cap\left\{\lambda_{m+1}, \lambda_{m+2}, \ldots, \lambda_{n}\right\}=\varnothing$ and $m<n$. Partition $\hat{X}=\left(\hat{X}_{1}, \hat{X}_{2}\right) \quad$ and $\hat{Y}=\left(\hat{Y}_{1}, \hat{Y}_{2}\right), \quad$ where $\hat{X}_{1}=\left(\hat{x}_{1}, \ldots \hat{x}_{m}\right)$, $\hat{X}_{2}=\left(\hat{x}_{m+1}, \ldots \hat{x}_{n}\right), \hat{Y}_{1}=\left(\hat{y}_{1}, \ldots \hat{y}_{m}\right)$ and $\hat{Y}_{2}=\left(\hat{y}_{m+1}, \ldots \hat{y}_{n}\right)$.

Then:

$\hat{Y}_{1}^{H} \hat{X}_{2}=0$

And:

$\hat{Y}_{1}^{H} A \hat{X}_{2}=0$

If, in addition, $A$ is real symmetric, then:

$\hat{Y}_{1}^{H} \hat{X}_{2}=0$ and $\hat{Y}_{1}^{H} A \hat{X}_{2}=0$

The following theorems establish the orthogonality relations between the eigenvectors for the matrix polynomial using its connection with the standard eigenvalues problem.

Theorem 2 (Ramadan and El-Sayed, 2010) A scalar $\lambda \in C$ is an eigenvalue of the matrix polynomial

$P(\lambda)=\lambda^{k} M_{k}+\lambda^{k-1} M_{k-1}+\cdots+\lambda M_{1}+M_{0} \quad$ with the corresponding right eigenvector $x$ and the left eigenvector $y$ if and only if $\lambda$ is an eigenvalue of the $k n \times k n$ matrix:

$$
A=\left(\begin{array}{ccccc}
0 & I & 0 & \cdots & 0 \\
0 & 0 & I & \cdots & 0 \\
\vdots & \vdots & \vdots & \vdots & \vdots \\
0 & 0 & 0 & \cdots & I \\
-M_{k}^{-1} M_{0}-M_{k}^{-1} M_{1}-M_{k}^{-1} M_{2} & \cdots-M_{k}^{-1} M_{k-1}
\end{array}\right)
$$

With the corresponding right eigenvector $\hat{x}$ and left eigenvector $\hat{y}$ such that:

$$
\begin{aligned}
\hat{x} & =\left(\begin{array}{c}
x \\
\lambda x \\
\lambda^{2} x \\
\vdots \\
\lambda^{k-1} x
\end{array}\right) \text { and } \\
\hat{y}= & \left(\begin{array}{c}
\left(\lambda^{k-1} M_{k}^{H}+\lambda^{k-2} M_{k-1}^{H}+\cdots+M_{1}^{H}\right) y \\
\left(\lambda^{k-2} M_{k}^{H}+\lambda^{k-3} M_{k-1}^{H}+\cdots+M_{2}^{H}\right) y \\
\left(\lambda^{k-3} M_{k}^{H}+\lambda^{k-4} M_{k-1}^{H}+\cdots+M_{3}^{H}\right) y \\
\vdots \\
\left(M_{k}^{H}\right) y
\end{array}\right)
\end{aligned}
$$


Theorem 3 (Ramadan and El-Sayed, 2010) (Orthogonality of the Eigenvectors of the Matrix Polynomial)

Let $\lambda_{1}, \lambda_{2}, \ldots, \lambda_{k n}$ be the eigenvalues of the $k n \times k n$ matrix polynomial $P(\lambda)=\lambda^{k} M_{k}+\lambda^{k-1} \quad M_{k-1}+\cdots+\lambda M_{1}+M_{0}$ and let $X$ and $Y$ be respectively the right and left eigenvector matrices.

Assume that $\left\{\lambda_{1}, \ldots, \lambda_{m}\right\} \cap\left\{\lambda_{m+1}, \ldots, \lambda_{k n}\right\}=\emptyset$. Partition $X=\left(X_{1}, X_{2}\right), Y=\left(Y_{1}, Y_{2}\right)$ and $\Lambda=\operatorname{diag}\left(\Lambda_{1}, \Lambda_{2}\right)$ where $X_{1}=$ $\left(x_{1}, \ldots, x_{m}\right), X_{2}=\left(x_{m+1}, \ldots, x_{k n}\right), Y_{1}=\left(y_{1}, \ldots, y_{m}\right)$ and $Y_{2}=$ $\left(y_{m+1}, \ldots, y_{k n}\right)$ with $\Lambda_{1}=\operatorname{diag}\left(\lambda_{1}, \ldots, \lambda_{m}\right)$ and $\Lambda_{2}=$ $\operatorname{diag}\left(\lambda_{m+1}, \ldots, \lambda_{k n}\right)$.

Then:

$\sum_{i=1}^{k-1}\left[\sum_{j=1}^{i}\left[\Lambda_{1}^{j} Y_{1}^{H} M_{k-i+j}\right]\right] X_{2} \Lambda_{2}^{k-i}-Y_{1}^{H} M_{0} X_{2}=0$

And:

$\sum_{i=1}^{k}\left[\sum_{j=1}^{i}\left[\Lambda_{1}^{j-1} Y_{1}^{H} M_{k-i+j}\right]\right] X_{2} \Lambda_{2}^{k-i}=0$

\section{PEAP for High Order Control Systems in a Time Delayed System in Multi-Input Case}

Write Equation (1.2) in the following form:

$$
P\left(\lambda_{i}\right) x_{i}=\left(\begin{array}{c}
\lambda_{i}^{k} M_{k}+\lambda_{i}^{k-1} M_{k-1} \\
+\ldots+\lambda_{i} M_{1}+M_{0}
\end{array}\right) x_{i}=0 i=1,2, \ldots, k n
$$

Or in the matrix form:

$$
M_{k} X \Lambda^{K}+M_{k-1} X \Lambda^{K-1}+\ldots+M_{0} X=0
$$

where, $X=\left(x_{1}, x_{2} \ldots, x_{k n}\right) \in C^{n \times k n}$ and $\Lambda=\operatorname{diag}\left(\lambda_{1}\right.$, $\left.\lambda_{2} \ldots, \lambda_{k n}\right) \in C^{k n \times k n}$ where $\lambda_{i}$ are all distinct. Where $\lambda_{1}, \ldots, \lambda_{k n}$ are the eigenvalues of matrix polynomial:

$$
P(\lambda)=\lambda^{k} M_{k}+\lambda^{k-1} M_{k-1}+\ldots+\lambda M_{1}+M_{0}
$$

Let us partition the $n \times k n$ right eigenvector matrix $X$, the $k n \times n$ left eigenvector matrix $Y^{H}$ and $k n \times k n$ eigenvalues matrix $\Lambda$ as follows:

$$
X=\left(X_{1}, X_{2}\right), Y^{H}=\left(\begin{array}{c}
Y_{1}^{H} \\
Y_{2}^{H}
\end{array}\right) \text { and } \Lambda=\operatorname{diag}\left(\Lambda_{1}, \Lambda_{2}\right)
$$

where, $X_{1}=\left(x_{1}, \ldots, x_{m}\right) X_{2}=\left(x_{m+1}, \ldots, x_{k n}\right), Y_{1}=\left(y_{1}, \ldots, y_{m}\right)$ and $Y_{2}=\left(y_{m+1}, \ldots, y_{k n}\right)$ with $\Lambda_{1}=\operatorname{diag}\left(\lambda_{1}, \ldots, \lambda_{m}\right)$ and $\Lambda_{2}=$ $\operatorname{diag}\left(\lambda_{m+1}, \ldots, \lambda_{k n}\right)$.

Given $m$ complex numbers $\mu_{1}, \mu_{2}, \ldots, \mu_{m}$ closed under complex conjugation, $m<n$ and a matrix $B \in R^{n \times m}$ with known time delay $\tau$, we are required to find $F_{1}$, $F_{2}, \ldots, F_{k} \in R^{n \times m}$ such that the modified matrix polynomial:

$$
\begin{aligned}
& P_{c}(\lambda, \tau)=\lambda^{k} M_{k}+\ldots+\lambda M_{1} \\
& +M_{0}-B\left(F_{1}^{T} \lambda^{k-1}+\ldots+F_{k-1}^{T} \lambda+F_{k}^{T}\right) e^{-\lambda \tau}
\end{aligned}
$$

has spectrum $\left\{\mu_{1}, \mu_{2}, \ldots, \mu_{m}, \lambda_{m+1}, \ldots, \lambda_{k n}\right\}$. PEAP for high-order linear systems in Multi-input case with time delay in which we use the matrices $F_{1}, F_{2}, \ldots, F_{k} \in R^{n \times m}$ to replace the eigenvalues $\left\{\lambda_{j}\right\}_{j=1}^{m}$ of the matrix polynomial $P(\lambda)=\lambda^{k} M_{k}+\lambda^{k-1} M_{k-1}+\cdots+\lambda M_{1}+M_{0}$ by $\left\{\mu_{j}\right\}_{j=1}^{m}$, while leaving the other eigenvalues unchanged. Next, we introduce the following theorem for solving PEAP of high order linear systems with time delay.

\section{Theorem 4}

Let the feedback matrices $\left\{F_{i}\right\}_{i=1}^{k-1}$ and $F_{k}$ be defined by:

$F_{i}=\sum_{j=1}^{i}\left[M_{k-i+j}^{T} \bar{Y}_{1} \Lambda_{1}^{j}\right] \varphi^{T}$,

$F_{k}=-M_{0}^{T} \bar{Y}_{1} \varphi^{T}, i=1,2 \ldots, k-1, \varphi \in C^{m \times m}$

Then for any choice of $\varphi$ we have:

$$
\begin{aligned}
& M_{k} X_{2} \Lambda_{2}^{k}+\ldots+M_{0} X_{2} \\
& -\left(B F_{1}^{T} X_{2} \Lambda_{2}^{k-1}+\ldots+B F_{k}^{T} X_{2}\right) e^{-\Lambda_{2} \tau}=0
\end{aligned}
$$

In words, this theorem assures us that any choice of $\varphi$ with the feedback matrices $\left\{F_{i}\right\}_{i=1}^{k}$ as in (3.6) guarantees that the last $k n-m$ eigenpairs $\left(\Lambda_{2}, X_{2}\right)$ of the matrix polynomial $P(\lambda)$ are also eigenpairs of the modified matrix polynomial $P_{c}(\lambda, \tau)$.

\section{Proof}

Our goal is to prove that:

$$
\begin{aligned}
& M_{k} X_{2} \Lambda_{2}^{k}+\ldots+M_{0} X_{2} \\
& -\left(B F_{1}^{T} X_{2} \Lambda_{2}^{k-1}+\ldots+B F_{k}^{T} X_{2}\right) e^{-\Lambda_{2} \tau}=0
\end{aligned}
$$

Expanding the left hand side of (3.8) by substituting (3.6) in (3.8), we obtain:

$$
\begin{aligned}
& M_{k} X_{2} \Lambda_{2}^{k}+\ldots+M_{0} X_{2} \\
& -B\left(\begin{array}{l}
\varphi \sum_{i=1}^{k-1} \sum_{j=1}^{i}\left[\Lambda_{1}^{j} Y_{1}^{H} M_{k-i+j}\right] \\
X_{2} \Lambda_{2}^{k-i}-\varphi Y_{1}^{H} M_{0} X_{2}
\end{array}\right) e^{-\Lambda_{2} \tau} \\
& =-B \varphi\left(\begin{array}{l}
\sum_{i=1}^{k-1} \sum_{j=1}^{i}\left[\Lambda_{1}^{j} Y_{1}^{H} M_{k-i+j}\right] \\
X_{2} \Lambda_{2}^{k-i}-Y_{1}^{H} M_{0} X_{2}
\end{array}\right) e^{-\Lambda_{2} \tau}
\end{aligned}
$$


Since $\left(X_{2}, \Lambda_{2}\right)$ is an eigenpair of the open loop pencil (3.2) we have:

$$
M_{k} X_{2} \Lambda_{2}^{k}+M_{k-1} X_{2} \Lambda_{2}^{k-1}+\ldots+M_{0} X_{2}=0
$$

And furthermore:

$\sum_{i=1}^{k-1}\left[\sum_{j=1}^{i}\left[\Lambda_{1}^{j} Y_{1}^{H} M_{k-i+j}\right]\right] X_{2} \Lambda_{2}^{k-i}-Y_{1}^{H} M_{0} X_{2}=0$

From theorem 3. Thus:

$$
\begin{aligned}
& M_{k} X_{2} \Lambda_{2}^{k}+\ldots+M_{0} X_{2} \\
& -\left(B F_{1}^{T} X_{2} \Lambda_{2}^{k-1}+\ldots+B F_{k}^{T} X_{2}\right) e^{-\Lambda_{2} \tau}=0
\end{aligned}
$$

The theorem is then proved.

In order to use theorem 4 to solve PEAP, we need to choose $\varphi$ which will change eigenvalues $\left\{\lambda_{j}\right\}_{j=1}^{m}$ of the matrix polynomial $P(\lambda)$ to $\left\{\mu_{j}\right\}_{j=1}^{m}$ in $P_{c}(\lambda, \tau)$, if that is possible. If there is such the matrix $\varphi^{T}$, then there exist an eigenvector matrix $Z \in C^{n \times m}$ such that $Z=\left(z_{1}, z_{2}, \ldots, z_{m}\right)$, $z_{j} \neq 0, j=1,2, \ldots, m$ and matrix $D=\operatorname{diag}\left(\mu_{1}, \mu_{2}, \ldots, \mu_{m}\right)$ and $e^{-D \tau}=\operatorname{diag}\left(e^{-\mu_{1} \tau}, e^{-\mu_{2} \tau}, \ldots, e^{-\mu_{m} \tau}\right)$ which are such that:

$M_{k} Z D^{k}+\ldots+M_{0} Z-\left(B F_{1}^{T} Z D^{k-1}+\ldots+B F_{k}^{T} Z\right) e^{-D \tau}=0$

Substituting $\left\{F_{i}\right\}_{i=1}^{k}$ form (3.6) in (3.13) and after rearranging, we obtain:

$$
\begin{aligned}
& M_{k} Z D^{k}+M_{k-1} Z D^{k-1}+\ldots+M_{0} Z \\
& =B \varphi\left(\begin{array}{l}
\sum_{i=1}^{k-1} \sum_{j=1}^{i}\left[\Lambda_{1}^{j} Y_{1}^{H} \mathrm{M}_{\mathrm{k}-\mathrm{i}+\mathrm{j}}\right] \\
Z D^{k-i}-Y_{1}^{H} M_{0} Z
\end{array}\right) e^{-D \tau}=B \varphi W^{H}
\end{aligned}
$$

Then:

$$
M_{k} Z D^{k}+M_{k-1} Z D^{k-1}+\ldots+M_{0} Z=B \Gamma
$$

where, $W^{H}=\left(\sum_{i=1}^{k-1} \sum_{j=1}^{i}\left[\Lambda_{1}^{j} Y_{1}^{H} \mathrm{M}_{\mathrm{k}-\mathrm{i}+\mathrm{j}}\right] Z D^{k-i}-Y_{1}^{H} M_{0} Z\right) e^{-D \tau}$ and $W \varphi^{H}=\Gamma^{H}$ is matrix that will depend on the scaling chosen for the eigenvectors in $Z$. To obtain $Z$, we choosing the matrix $\Gamma$ in the following form $\Gamma=\left(\gamma_{1}\right.$, $\left.\gamma_{2}, \ldots, \gamma_{m}\right)$. Then the equation (3.15) becomes:

$$
M_{k} Z D^{k}+M_{k-1} Z D^{k-1}+\ldots+M_{0} Z=B\left(\gamma_{1}, \gamma_{2}, \ldots, \gamma_{m}\right)
$$

We can solve for each of the eigenvectors $z_{j}$ using the equations in such a way that $\mu_{j}=\bar{\mu}_{i}$ implies $\gamma_{j}=\bar{\gamma}_{i}$ for $i=1,2, \ldots, m$ and solve for $z_{1}, \ldots, z_{m}$ :
$\left(M_{k} \mu_{j}^{k}+M_{k-1} \mu_{j}^{k-1}+\ldots+M_{0}\right) z_{j}=B \gamma_{j}, j=1,2, \ldots, m$

So, we computed the eigenvectors $Z$ and hence we computed the matrix $W$ from:

$W^{H}=\left(\sum_{i=1}^{k-1} \sum_{j=1}^{i}\left[\Lambda_{1}^{j} Y_{1}^{H} M_{k-i+j}\right] Z D^{k-i}-Y_{1}^{H} M_{0} Z\right) e^{-D \tau}$

We solve the $m \times m$ square linear system for $\varphi$ :

$W \varphi^{H}=\left(\gamma_{1}, \gamma_{2}, \ldots, \gamma_{m}\right)^{H}$

Finally, determine the control gain matrices $F_{i}, i=$ $1,2, \ldots, k$ are obtained from (3.6).

\section{PEAP for High Order Control Systems in a Time Delayed System in Single-Input Case}

For single-input control application, the system (1.1) is modified by applying a controlling force $b u(t-\tau)$ as:

$$
\begin{aligned}
& M_{k} \frac{d^{k}}{d t^{k}} v(t)+M_{k-1} \frac{d^{k-1}}{d t^{k-1}} v(t) \\
& +\ldots+M_{1} \frac{d}{d t} v(t)+\mathrm{M}_{0} \mathrm{v}(\mathrm{t})=b u(t-\tau)
\end{aligned}
$$

where, $b \in R^{n \times 1}$, ( $b$ is the control vector) and control force $u(t-\tau)$, is defined as:

$$
\begin{aligned}
& u(t-\tau)=f_{1}^{T} \frac{d^{k-1}}{d t^{k-1}} v(t-\tau) \\
& +\ldots+f_{k-1}^{T} \frac{d}{d t} v(t-\tau)+f_{k}^{T} v(t-\tau)
\end{aligned}
$$

Such that, $f_{1}, f_{2}, \ldots, f_{k}$ vectors, leads to the closed loop system:

$$
\begin{aligned}
& M_{k} \frac{d^{k}}{d t^{k}} v(t)+M_{k-1} \frac{d^{k-1}}{d t^{k-1}} v(t)+\ldots+M_{1} \frac{d}{d t} v(t)+M_{0} v(t) \\
& =b\left(f_{1}^{T} \frac{d^{k-1}}{d t^{k-1}} v(t-\tau)+\ldots+f_{k-1}^{T} \frac{d}{d t} v(t-\tau)+f_{k}^{T} v(\mathrm{t}-\tau)\right)
\end{aligned}
$$

where, $\tau$ is the known time delay with the separation of variables $v(t)=x e^{\lambda t}$, to the problem of finding the eigenvalues of the modified matrix polynomial:

$$
\begin{aligned}
& P_{c}(\lambda, \tau)=\lambda^{k} M_{k}+\ldots+\lambda M_{1} \\
& +M_{0}-b\left(f_{1}^{T} \lambda^{k-1}+\ldots+f_{k-1}^{T} \lambda+f_{k}^{T}\right) e^{-\lambda \tau}
\end{aligned}
$$

PEAP with the time delayed system (4.4) is to find the control vectors $f_{1}, f_{2}, \ldots, f_{k}$ such that $\operatorname{det}\left(P_{c}\left(\lambda_{i, \tau}\right)\right)=0$, 
for $i=1,2, \ldots, k n$, has the desired eigenvalues $\left\{\mu_{1}\right.$, $\left.\mu_{2}, \ldots, \mu_{m}, \lambda_{m+1}, \ldots, \lambda_{k n}\right\}$. That it contains $m$ numbers of newly assign need desired e igenvalues $\left.\mu_{1}, \mu_{2}, \ldots, \mu_{m}\right\}$ without affecting the remaining eigenvalues $\left\{\lambda_{m+1}, \ldots, \lambda_{k n}\right\}$ of the open loop system (1.1).

\section{Theorem 5}

Let the feedback vectors $\left\{f_{i}\right\}_{i=1}^{k-1}$ and $f_{k}$ be defined by:

$$
\begin{aligned}
& f_{i}=\sum_{j=1}^{i}\left[M_{k-i+j}^{T} \bar{Y}_{1} \Lambda_{1}^{j}\right] \varphi^{T}, f_{k}=-M_{0}^{T} \bar{Y}_{1} \varphi^{T}, \\
& i=1,2 \ldots, k-1 . \varphi \in C^{1 \times m}
\end{aligned}
$$

Then for any choice of $\varphi$ the following relation holds:

$$
\begin{aligned}
& M_{k} X_{2} \Lambda_{2}^{k}+\ldots+M_{0} X_{2} \\
& -\left(b f_{1}^{T} X_{2} \Lambda_{2}^{k-1}+\ldots+b f_{k}^{T} X_{2}\right) e^{-\Lambda_{2} \tau}=0
\end{aligned}
$$

\section{Proof}

Our goal is to prove that:

$$
\begin{aligned}
& M_{k} X_{2} \Lambda_{2}^{k}++\ldots+M_{0} X_{2} \\
& -\left(b f_{1}^{T} X_{2} \Lambda_{2}^{k-1}+\ldots+b f_{k}^{T} X_{2}\right) e^{-\Lambda_{2} \tau}=0
\end{aligned}
$$

Expanding the left hand side of (4.7) by substituting (4.5) in (4.7), we obtain:

$$
\begin{aligned}
& M_{k} X_{2} \Lambda_{2}^{k}+\ldots+M_{0} X_{2}-b\left(\begin{array}{c}
\varphi \sum_{i=1}^{k-1} \sum_{j=1}^{i}\left[\Lambda_{1}^{j} Y_{1}^{H} M_{k-i+j}\right] \\
X_{2} \Lambda_{2}^{k-i}-\varphi Y_{1}^{H} M_{0} X_{2}
\end{array}\right) e^{-\Lambda_{2} \tau} \\
& =-b \varphi\left(\sum_{i=1}^{k-1} \sum_{j=1}^{i}\left[\Lambda_{1}^{j} Y_{1}^{H} M_{k-i+j}\right] X_{2} \Lambda_{2}^{k-i}-Y_{1}^{H} M_{0} X_{2}\right) e^{-\Lambda_{2} \tau}
\end{aligned}
$$

Since $\left(X_{2}, \Lambda_{2}\right)$ is an eigenpair of the open loop pencil we have:

$$
M_{k} X_{2} \Lambda_{2}^{k}+M_{k-1} X_{2} \Lambda_{2}^{k-1}+\ldots+M_{0} X_{2}=0
$$

And furthermore:

$$
\sum_{i=1}^{k-1}\left[\sum_{j=1}^{i}\left[\Lambda_{1}^{j} Y_{1}^{H} M_{k-i+j}\right]\right] X_{2} \Lambda_{2}^{k-i}-Y_{1}^{H} M_{0} X_{2}=0
$$

From theorem 3. Thus:

$$
\begin{aligned}
& M_{k} X_{2} \Lambda_{2}^{k}+\ldots+M_{0} X_{2} \\
& -\left(b f_{1}^{T} X_{2} \Lambda_{2}^{k-1}+\ldots+b f_{k}^{T} X_{2}\right) e^{-\Lambda_{2} \tau}=0
\end{aligned}
$$

The theorem is then proved.
From theorem 5, we need to choose $\varphi$ which will change eigenvalues $\left\{\lambda_{j}\right\}_{j=1}^{m}$ of the matrix polynomial $P(\lambda)$ to $\left\{\mu_{j}\right\}_{j=1}^{m}$ in $P_{c}(\lambda, \tau)$, if that is possible. If there is such the matrix $\varphi^{T}$, then there exist an eigenvector matrix $Z \in C^{n \times m}$ such that $Z=\left(z_{1}, z_{2}, \ldots, z_{m}\right), z_{j} \neq 0, j=1,2, \ldots, m$ and matrix $D=\operatorname{diag}\left(\mu_{1}, \quad \mu_{2}, \ldots, \mu_{m}\right) \quad$ and $e^{-D \tau}=\operatorname{diag}\left(e^{-\mu_{1} \tau}, e^{-\mu_{2} \tau}, \ldots, e^{-\mu_{m} \tau}\right)$ which are such that:

$M_{k} Z D^{k}+\ldots+M_{0} Z-\left(b f_{1}^{T} Z D^{k-1}+\ldots+b f_{k}^{T} Z\right) e^{-D \tau}=0$

Substituting $\left\{f_{i}\right\}_{i=1}^{k}$ form (4.5) in (4.12) and after rearranging, we obtain:

$M_{k} Z D^{k}+\ldots+M_{0} Z$
$=b \varphi\left(\begin{array}{l}\sum_{i=1}^{k-1} \sum_{j=1}^{i}\left[\Lambda_{1}^{j} Y_{1}^{H} M_{k-i+j}\right] \\ Z D^{k-i}-Y_{1}^{H} M_{0} Z\end{array}\right) e^{-D \tau}=b \varphi W^{H}$

Then:

$M_{k} Z D^{k}+M_{k-1} Z D^{k-1}+\ldots+M_{0} Z=b C$

where, $W^{H}=\left(\sum_{i=1}^{k-1} \sum_{j=1}^{i}\left[\Lambda_{1}^{j} Y_{1}^{H} M_{k-i+j}\right] Z D^{k-i}-Y_{1}^{H} M_{0} Z\right) e^{-D \tau}$ and $W \varphi^{H}=C^{H}$ is matrix that will depend on the scaling chosen for the eigenvectors in $Z$. To obtain $Z$, we choosing the vector $C$ in the following $C=(1,1, \ldots, 1)$. Then the equation (4.14) becomes:

$M_{k} Z D^{k}+M_{k-1} Z D^{k-1}+\ldots+M_{0} Z=b(1,1, \ldots, 1)$

We can solve for each of the eigenvectors $z_{j}$ :

$\left(M_{k} \mu_{j}^{k}+M_{k-1} \mu_{j}^{k-1}+\ldots+M_{0}\right) z_{j}=b, j=1,2, \ldots, m$

So, we computed the eigenvectors $Z$ and hence we computed the matrix $W$ from:

$W^{H}=\left(\sum_{i=1}^{k-1} \sum_{j=1}^{i}\left[\Lambda_{1}^{j} Y_{1}^{H} M_{k-i+j}\right] Z D^{k-i}-Y_{1}^{H} M_{0} Z\right) e^{-D \tau}$

We solve the $m \times 1$ linear system for $\varphi$ :

$W \varphi^{H}=(1,1, \ldots, 1)^{T}$

Finally, determine the control vectors $f_{i}, i=1,2, \ldots, k$ are obtained from (4.5). 


\section{Numerical Examples}

\section{Example (1) (Multi-Input Case)}

Let the randomly matrices $M_{3}, M_{2}, M_{1}$ and $M_{0}$ (size 4) as follows:

$$
\begin{aligned}
& M_{3}=\left[\begin{array}{llll}
0.9501 & 0.8913 & 0.8214 & 0.9218 \\
0.2311 & 0.7621 & 0.4447 & 0.7382 \\
0.6068 & 0.4565 & 0.6154 & 0.1763 \\
0.4860 & 0.0185 & 0.7919 & 0.4057
\end{array}\right] \\
& M_{2}=\left[\begin{array}{llll}
0.3046 & 0.3028 & 0.3784 & 0.4966 \\
0.1897 & 0.5417 & 0.8600 & 0.8998 \\
0.1934 & 0.1509 & 0.8537 & 0.8216 \\
0.6822 & 0.6979 & 0.5936 & 0.6449
\end{array}\right] \\
& M_{1}=\left[\begin{array}{llll}
0.4451 & 0.8462 & 0.8381 & 0.8318 \\
0.9318 & 0.5252 & 0.0196 & 0.5028 \\
0.4660 & 0.2026 & 0.6813 & 0.7095 \\
0.4186 & 0.6721 & 0.3795 & 0.4289
\end{array}\right] \\
& M_{0}=\left[\begin{array}{llll}
0.9355 & 0.0579 & 0.1389 & 0.2722 \\
0.9169 & 0.3529 & 0.2028 & 0.1988 \\
0.4103 & 0.8132 & 0.1987 & 0.0153 \\
0.8936 & 0.0099 & 0.6038 & 0.7468
\end{array}\right]
\end{aligned}
$$

And a randomly matrix $B$ (the control matrix), is:

$$
B=\left[\begin{array}{cc}
8.1797 \mathrm{e}-001 & 8.1797 \mathrm{e}-001 \\
3.4197 \mathrm{e}-001 & 2.8973 \mathrm{e}-001 \\
3.4119 \mathrm{e}-001 & 5.3408 \mathrm{e}-001 \\
7.2711 \mathrm{e}-001 & 3.0929 \mathrm{e}-001
\end{array}\right]
$$

And $\tau=0.1$ eigenvalues of $P(\lambda)=$ $M_{3} \lambda^{3}+M_{2} \lambda^{2}+M_{1} \lambda+M_{0}$ are: $\lambda_{1,2}=1.1687 \pm 0.8481 i, \lambda_{3,4}=$ $0.0375 \pm 0.9101 i, \lambda_{5,6}=-0.2044 \pm 0.5375 i, \lambda_{7}=0.1275, \lambda_{8}$ $=0.6132, \lambda_{9}=0.9085, \lambda_{10}=-0.9321, \lambda_{11}=-1.6155, \lambda_{12}$ $=-2.1822$.

Now, we reassign the first two $m=2$ eigenvalues $\lambda_{1,2}$ $=1.1687 \pm 0.8481 i$ to the conjugate pair $\mu_{1,2}=-1 \pm i$.

From theorem 3, we obtain $\Lambda_{1}=$ $\operatorname{diag}(1.1687+0.8481 i, 1.1687-0.8481 i)$ :

$$
Y_{1}^{H}=\left[\begin{array}{cc}
-0.1014+0.6844 i & -0.1014-0.6844 i \\
0.0808-0.3962 i & 0.0808+0.3962 i \\
-0.6505-0.3211 i & -0.6505+0.3211 i \\
0.7606-0.0701 i & 0.7606+0.0701 i
\end{array}\right]
$$

And $D=\operatorname{diag}(-1+i,-1-i)$. By following the procedure in the section 3 and assume that:

$$
\Gamma=\left(\gamma_{1}, \gamma_{2}\right)=\left[\begin{array}{ll}
0.83850-0.37041 i & 0.56807-0.70274 i \\
0.83850+0.37041 i & 0.56807+0.70274 i
\end{array}\right]
$$

We compute:

$$
W=\left[\begin{array}{cc}
0.0350+0.1648 i & -0.0791-0.3293 i \\
-0.0791+0.3293 i & 0.0350-0.1648 i
\end{array}\right]
$$

And:

$$
\varphi=\left[\begin{array}{ll}
1.0117-1.7874 i & 3.2719-1.4419 i \\
1.0117+1.7874 i & 3.2719+1.4419 i
\end{array}\right]
$$

From which we compute the feedback matrices, $F_{1}$, $\mathrm{F}_{2}$ and $F_{3}$ in view of (3.6):

$$
\begin{gathered}
F_{1}=\left[\begin{array}{cc}
-1.8746 & -3.4758 \\
-0.7359 & -3.0500 \\
-0.8857 & -0.3800 \\
-1.5998 & -1.4270
\end{array}\right], \\
F_{2}=\left[\begin{array}{cc}
-1.8799 & -2.9836 \\
-0.7359 & 1.3408 \\
0.6590 & 0.9684 \\
-0.5921 & -1.2031
\end{array}\right]
\end{gathered}
$$

And

$$
F_{3}=\left[\begin{array}{cc}
-0.4984 & -2.3275 \\
-0.2848 & 2.2204 \\
-0.9992 & -2.4381 \\
-0.9263 & -3.4316
\end{array}\right]
$$

Once the control gain matrices are obtained, by computing:

$$
\operatorname{det}\left(P_{c}\left(\lambda_{i}, \tau\right)\right)=\operatorname{det}\left(\begin{array}{l}
\lambda_{i}^{3} M_{3}+\lambda_{i}^{2} M_{2}+\lambda_{i} M_{1}+M_{0}-B \\
\left(F_{1}^{T} \lambda_{i}^{2}+F_{2}^{T} \lambda_{i}+F_{3}^{T}\right) e^{-\lambda_{i} \tau}
\end{array}\right)
$$

For $i=1,2, \ldots, 12$. Where $\lambda_{i} \in\left\{\mu_{1}, \mu_{2}, \lambda_{3}, \lambda_{4} \ldots, \lambda_{12}\right\}$, it is verified that $\left|\operatorname{det}\left(P_{c}\left(\lambda_{i}, \tau\right)\right)\right|<10^{-12}$ validating the desired partial eigenvalue assignment.

\section{Example (2) (Single-Input Case)}

Let the randomly matrices $M_{3}, M_{2}, M_{1}$ and $M_{0}$ (size 4) as in example 1 and a randomly vector $b$ (the control vector):

$$
b=\left[\begin{array}{l}
0.8180 \\
0.6602 \\
0.3420 \\
0.2897
\end{array}\right] \text { and } \tau=0.1
$$


Eigenvalues of $P(\lambda)=M_{3} \lambda^{3}+M_{2} \lambda^{2}+M_{1} \lambda+M_{0}$ are $: \lambda_{1,2}=$ $1.1687 \pm 0.8481 i, \quad \lambda_{3,4}=0.0375 \pm 0.9101 i, \quad \lambda_{5,6}=-$ $0.2044 \pm 0.5375 i, \lambda_{7}=0.1275, \lambda_{8}=0.6132, \lambda_{9}=0.9085$, $\lambda_{10}=-0.9321, \lambda_{11}=-1.6155, \lambda_{12}=-2.1822$.

Now, we reassign the first two $m=2$ eigenvalues $\lambda_{1,2}$ $=1.1687 \pm 0.8481 i$ to the conjugate pair $\mu_{1,2}=-1 \pm i$.

From theorem 3, we obtain $\Lambda_{1}=$ $\operatorname{diag}(1.1687+0.8481 i, 1.1687-0.8481 i)$ :

$$
Y_{1}^{H}=\left[\begin{array}{cc}
-0.1014+0.6844 i & -0.1014-0.6844 i \\
0.0808-0.3962 i & 0.0808+0.3962 i \\
-0.6505-0.3211 i & -0.6505+0.3211 i \\
0.7606-0.0701 i & 0.7606+0.0701 i
\end{array}\right]
$$

And $D=\operatorname{diag}(-1+i,-1-i)$. By the procedure in the section 4 we compute:

$$
\begin{aligned}
& W=\left[\begin{array}{ll}
0.0198-0.0937 i & 0.0887+0.0889 i \\
0.0887-0.0889 i & 0.0198+0.0937 i
\end{array}\right] \\
& \text { and } \varphi=\left[\begin{array}{l}
10.4513-0.7334 i \\
10.4513+0.7334 i
\end{array}\right]
\end{aligned}
$$

We compute the feedback vectors, $f_{1}, f_{2}$ and $f_{3}$ in view of (4.5):

$$
f_{1}=\left[\begin{array}{c}
-7.9192 \\
-10.5668 \\
1.8435 \\
0.0530
\end{array}\right], f_{2}=\left[\begin{array}{c}
-5.7201 \\
1.5189 \\
1.6624 \\
-2.9669
\end{array}\right] \text { and } f_{3}=\left[\begin{array}{c}
-8.3155 \\
10.9586 \\
-6.8112 \\
-11.4977
\end{array}\right]
$$

Once the control gain vectors are obtained, by computing:

$$
\operatorname{det}\left(P_{c}\left(\lambda_{i}, \tau\right)\right)=\operatorname{det}\left(\begin{array}{l}
\lambda_{i}^{3} M_{3}+\lambda_{i}^{2} M_{2}+\lambda_{i} M_{1}+M_{0}-b \\
\left(f_{1}^{T} \lambda_{i}^{2}+f_{2}^{T} \lambda_{i}+f_{3}^{T}\right) e^{-\lambda_{i} \tau}
\end{array}\right)
$$

For $i=1,2, \ldots, 12$. Where $\lambda_{i} \in\left\{\mu_{1}, \mu_{2}, \lambda_{3}, \lambda_{4} \ldots, \lambda_{12}\right\}$, it is verified that $\left|\operatorname{det}\left(P_{c}\left(\lambda_{i}, \tau\right)\right)\right|<10^{-12}$ validating the desired partial eigenvalue assignment.

\section{Conclusion}

We derived a solution to PEAP for high-order linear systems (1.4) in both multi-input and single-input case by using orthogonality relations between eigenvectors for matrix polynomial $P(\lambda)$. The approach needs the knowledge of only a few eigenvalues and with corresponding left eigenvectors of matrix polynomial $P(\lambda)$ without turning high order systems into first order form. When $\tau=0$ is a special case of our results see the reference (Ramadan and El-Sayed, 2010).

\section{Acknowledgement}

This project was supported by the Deanship of Scientific Research at Prince Sattam Bin Abdulaziz University under the research project \# 2014/01/2293.

\section{Author's Contributions}

Ehab A. El-Sayed: Participated in all experiments, coordinated the data-analysis and contributed to the writing of the manuscript.

Eid E. EI Behady: Designed the research plan and organized the study and contributed to the writing of the manuscript.

\section{Ethics}

Department of Mathematics, College of Science and Humanitarian Studies,Prince Sattam Bin Abdulaziz University, Al-Kharj, Saudi Arabia.

\section{References}

Arévalo, C. and P. Lötstedt, 1995. Improving the accuracy of BDF methods for index 3 differentialalgebraic equations. BIT Numer. Math., 36: 297-308. DOI: $10.1007 / \mathrm{BF} 01732606$

De Boor, C. and H. Kreiss, 1986. On the condition of the linear systems associated with discretized BVPs of ODEs. SIAM J. Numer. Anal., 23: 936-939. DOI: $10.1137 / 0723061$

Dedieu, J.P. and F. Tisseur, 2001. Structured pseudospectra for polynomial eigenvalue problems, with applications. SIAM J. Matrix Anal. Applic., 29: 187-208. DOI: $10.1137 / \mathrm{S} 0895479800371451$

Dedieu, J.P. and F. Tisseur, 2003. Perturbation theory for homogeneous polynomial eigenvalue problems. Linear Algebra Applic., 358: 71-94. DOI: $10.1016 / \mathrm{S} 0024-3795(01) 00423-2$

Duan, G.R., 2005. Parametric approaches for eigenstructure assignment in high-order linear systems. Int. J. Control Automat. Syst., 3: 419-429.

Mackey, D.S., N. Mackey, C. Mehl and V. Mehrmann, 2006. Vector spaces of linearizations for matrix polynomials, SIAM J. Matrix Anal. Applic., 29: 143-159. DOI: $10.1137 / 050628350$

Prattand, J.M. and K.V. Singh, 2009. Quadratic partial eigenvalue assignment problem with time delay for active vibration control. J. Phys. Conf. Series, 181: 012092-012092.

DOI: $10.1088 / 1742-6596 / 181 / 1 / 012092$

Ramadan, M.A. and E.A. El-Sayed, 2010. Partial eigenvalue assignment problem of high order control systems using orthogonality relations. Comput. Math. Applic., 59: 1918-1928.

DOI: 10.1016/j.camwa.2009.07.063 
Sand, J., 2002. On implicit Euler for high-order highindex DAEs. Applied Numer. Math., 42: 411-424. DOI: $10.1016 / \mathrm{S} 0168-9274(01) 00164-7$

Sarkissian, D.R., 2001. Theory and computations of partial eigenvalue and eigenstructure assignment problems in matrix second-order and distributedparameter systems, PhD Thesis, Northern Illinois University.

Singh, K.V., R. Dey and B.N. Datta, 2014. Partial eigenvalue assignment and its stability in a time delayed system. Mechanical Syst. Signal Process., 42: 247-257. DOI: 10.1016/j.ymssp.2013.08.028
Wang, X.T. and L. Zhang, 2013. Partial eigenvalue assignment of high order systems with time delay. Linear Algebra Applic., 438: 2174-2187. DOI: $10.1016 /$ j.laa.2012.10.011

Wang, X.T. and L. Zhang, 2014. Partial eigenvalue assignment for high order system by multi-input control. Mechanical Syst. Signal Process., 42: 129-136. DOI: 10.1016/j.ymssp.2013.06.026 